\title{
Persistência do Canal Arterial e Endarterite da Artéria Pulmonar
}

\author{
Ivan Romero Rivera, Valdir A mbrósio Moisés, A nselmo Cotrim Brandão, Celia Camelo Silva, \\ José Lázaro Andrade, Antonio Carlos Carvalho
}

São Paulo, SP

\begin{abstract}
Descrevemos uma paciente de sete anos com diagnóstico ecocardiográfico e confirmação cirúrgica de endarterite de artéria pulmonar, citada como complicação da persistência do canal arterial e ilustramos a necessidade de ressecção cirúrgica completa do processo infeccioso para evitar outras complicações, como embolia pulmonar.
\end{abstract}

\section{Patent Ductus Arteriosus and Pulmonary Artery Endarteritis}

We describe a seven year old girl with echocardiographic diagnosis of pulmonary artery endarteritis as a complication of a patent ductus arteriosus, confirmed at surgery. This case illustrates the necessity of complete surgical resection of the infectious source as a way to avoid other complications such as pulmonary embolism.

Arq Bras Cardiol, volume 69 (n 5), 335-338, 1997

A endarterite de artéria pulmonar é uma entidade rara, com poucos casos relatados na literatura, a maioria das vezes presente em usuários de drogas endovenosas ${ }^{1}$, ou como complicação de shunts intracavitários ${ }^{2,3}$ ou persistência de canal arterial (PCA) ${ }^{4-6,8}$, ou ainda, como complicação secundária a procedimentos invasivos intracardíacos ${ }^{7}$.

Apresentamos um caso de endarterite de artéria pulmonar em paciente com PCA, diagnosticado mediante ecocardiografia bidimensional e submetida a tratamento cirúrgico com boa evolução.

\section{Relato do Caso}

Paciente de sete anos, branca, sexo feminino, $17 \mathrm{~kg}$ de peso, com antecedente pregresso de ausculta de sopro desde a infância, que começou a apresentar febre diária de $40,2^{\circ} \mathrm{C}$ oito meses antes da nossa avaliação, tendo nessa época feito uso de Bactrim durante sete dias sem melhora do quadro. Três meses depois do início da febre começou a apresentar anemia importante, sendo que 25 dias antes de ser transferida para a nossa instituição foi feita hemotransfusão e realizada tomografia abdominal, constatando-

Escola Paulista de Medicina - UNIFESP, São Paulo

Correspondência: Antonio C. Carvalho - Av. Jandira 731/23 - 04080-004 - São Paulo, SP

Recebido para publicação em 14/8/97

Aceito em 16/9/97 se hepatomegalia. $\mathrm{Na}$ época começou a apresentar tosse seca e hematúria. Ainda, três dias antes da nossa avaliação, foi realizado ecocardiograma (ECO) que diagnosticou persistência do canal arterial e endocardite de valva pulmonar.

A paciente ingressou no hospital em regular estado geral, descorada ++++ , hidratada, febril $\left(39^{\circ} \mathrm{C}\right)$, anictérica, acianótica, dispnéica, taquicárdica. Apresentava ictus visível e palpável no $6^{\circ}$ espaço intercostal esquerdo/linha axilar média; frêmito sistólico palpável no precórdio e ausculta de $3^{\mathrm{a}} \mathrm{e} 4^{\mathrm{a}}$ bulhas, sopro contínuo $+++/ 6$ na região paraesternal esquerda alta e $2^{\mathrm{a}}$ bulha hiperfonética. $\mathrm{O}$ abdômen era globoso, normotenso, doloroso à palpação, com ruídos hidroaéreos presentes, fígado palpável a $6 \mathrm{~cm}$ e baço a aproximadamente $9 \mathrm{~cm}$ do rebordo costal. Os membros inferiores apresentavam petéquias e edema + . Não havia sinais meníngeos e a otoscopia era normal. Realizado hemograma que mostrou: hemácias: $2,4 \times 10^{6} / \mathrm{mm}^{3}$; hemoglobina de 6,8g/dl; hematócrito de $21 \%$; plaquetas de $90000 / \mathrm{mm}^{3}$, VHS de $90 \mathrm{~mm}$ na $1^{\text {a }}$ hora e leucócitos de 10700 , sem neutrofilia.

O eletrocardiograma mostrou taquicardia sinusal com freqüência cardíaca (FC) de 130bpm, sobrecarga biatrial e ventricular esquerda e alterações difusas da repolarização ventricular(fig. 1), e à radiografia de tórax, cardiomegalia ++ e hiperfluxo pulmonar bilateral.

O ECO mostrou situs solitus, concordância atrioventricular e ventrículo-arterial, septos interatrial e interventricular íntegros, dilatação discreta do átrio esquerdo, dilatação e hipertrofia discretas do ventrículo direito, função sistólica normal de ambos os ventrículos, valvas 
atrioventriculares normais com refluxos discretos, valva pulmonar com vegetação de aproximadamente $4 \mathrm{~mm}$ aderida a sua face arterial e refluxo valvar moderado, PCA medindo $10 \mathrm{~mm}$ de diâmetro com fluxo esquerda-direita ao estudo com Doppler e mapeamento de fluxo a cores e presença de múltiplas vegetações no interior do tronco e artérias pulmo- nares, sendo a maior de $18 \mathrm{~mm}$ (fig. 2). A pressão sistólica da artéria pulmonar foi estimada em $90 \mathrm{mmHg}$.

As hemoculturas evidenciaram crescimento de Streptococcus Milleri, sensível a penicilina. Foi iniciada antibioticoterapia com penicilina cristalina na dose de $150000 \mathrm{UI} / \mathrm{kg} / \mathrm{dia}$ e amicacina na dose de $15 \mathrm{mg} / \mathrm{kg} / \mathrm{dia}$.

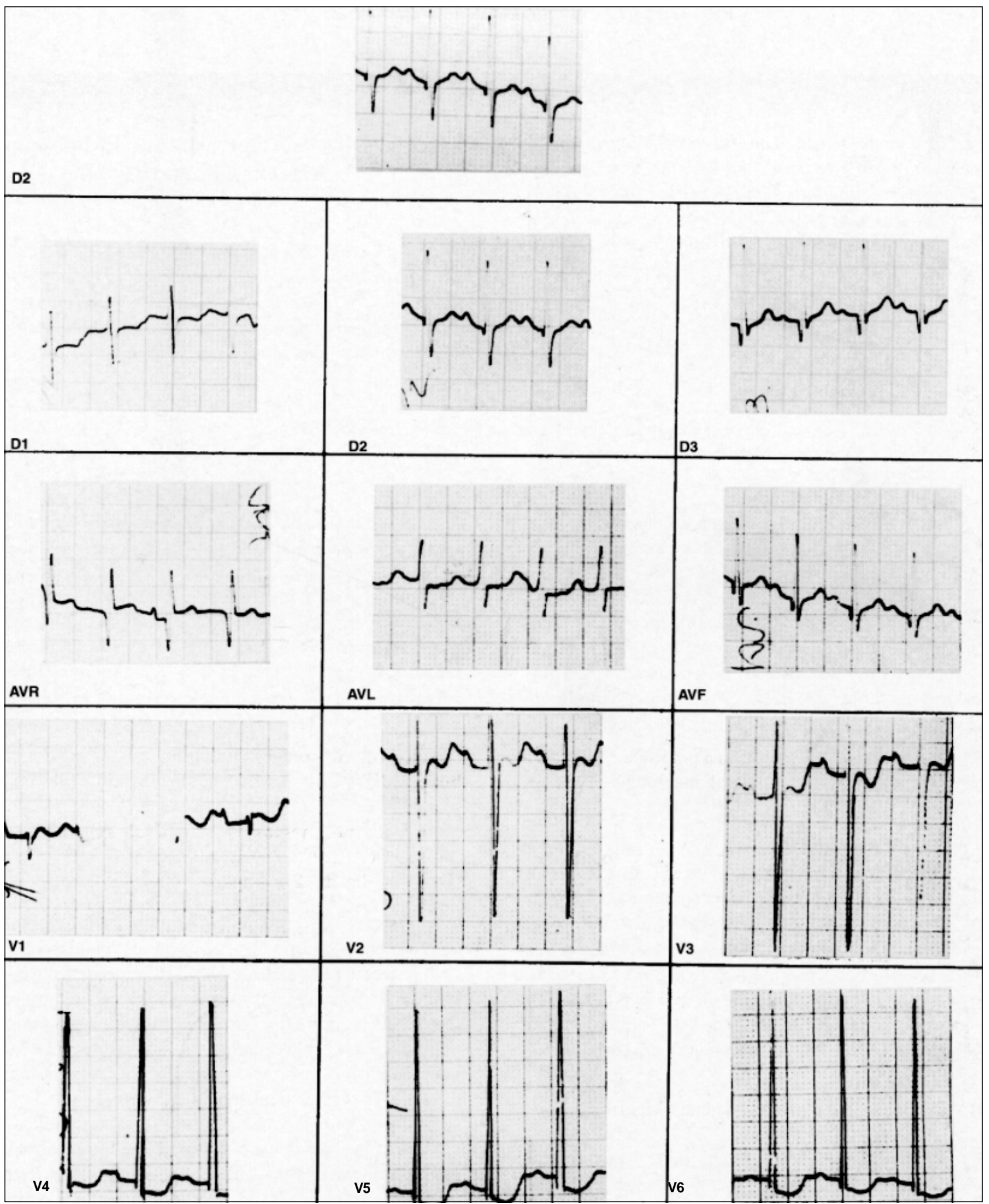

Fig. 1 - Eletrocardiograma demonstrando sobrecarga biatrial e do ventrículo esquerdo e alterações da repolarização ventricular. 


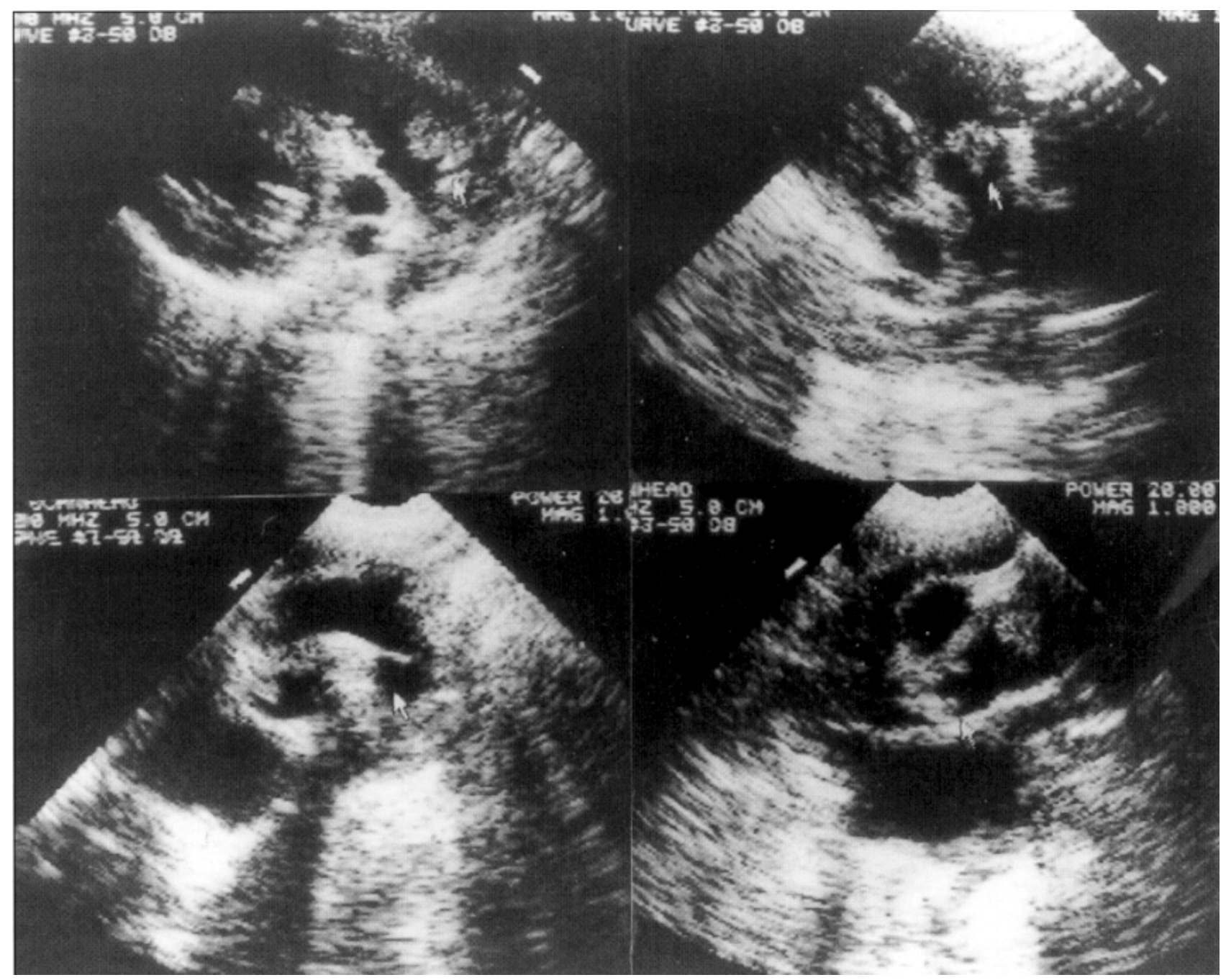

Fig. 2 - Dados ecocardiográficos mostrando nas figuras superiores: múltiplas vegetações no interior do tronco da artéria pulmonar e aderidas à valva pulmonar. Abaixo à esquerda: no canal arterial e no ramo esquerdo e à direita no interior do ramo direito da artéria pulmonar.

Após a introdução do antibiótico a paciente começou a apresentar melhora do estado geral, mantendo-se porém, ainda febril.

Realizada cintilografia pulmonar de perfusão que mostrou áreas de hipoconcentração anômala do radioindicador nos segmentos póstero-basal do lobo inferior, anterior do lobo superior do pulmão esquerdo e segmento pósterobasal do lobo inferior do pulmão direito.

A partir do $14^{\circ}$ dia as hemoculturas foram estéreis, sendo suspensa a amicacina e introduzida gentamicina na dose de $5 \mathrm{mg} / \mathrm{kg} / \mathrm{dia}$. Foi realizado cateterismo cardíaco para investigação de aneurisma micótico em artéria pulmonar, com resultado negativo.

A paciente foi submetida a tratamento cirúrgico 37 dias após a internação, em bom estado geral e afebril. Pela presença de infecção latente no local, não foi realizada ressecção do canal arterial, que foi fechado, mediante ligadura das extremidades aórtica e pulmonar e da sua região central.

Apresentou boa evolução imediata, em uso de penicilina e gentamicina. Um dia após a cirurgia apresentou três episódios de taquipnéia, cianose, hipertonia de membros e bradicardia (FC 40-50bpm), sendo diagnosticado embolia pulmonar. OECO mostrou ainda vegetações na artéria pulmonar e a ultra-sonografia pulmonar espessamento pleural à esquerda sem derrame. Foi indicada nova intervenção cirúrgica, realizada 12 dias após a $1^{\mathrm{a}}$ cirurgia, com abertura do tronco pulmonar, limpeza do local e extração de todas as vegetações. O ECO pós-operatório mostrou tronco e artérias pulmonares livres de vegetações.

Apresentou boa evolução pós-operatória e 10 dias depois foi dada de alta em TF I, afebril, em uso de digoxina $(10 \mu \mathrm{g} / \mathrm{kg} / \mathrm{dia})$. Na última avaliação, um mês após a cirurgia, encontrava-se assintomática e sem medicação.

\section{Discussão}

Apesar da PCA ser freqüentemente citada como fator de risco para o desenvolvimento de endocardite infecciosa, existem poucos relatos da literatura que documentem esta associação $^{4-6,8}$. 
Vargas-Barron e col $^{4}$ descreveram, em 1985, os sinais ecocardiográficos para o diagnóstico de endarterite da artéria pulmonar secundária à PCA, provavelmente $\mathrm{o} 1^{\circ}$ relato ecocardiográfico da doença.

Nosso caso é interessante não só pela evolução prolongada da doença, mas também pela exuberância das lesões, tanto na valva pulmonar como no tronco e ramos da artéria pulmonar. A maioria dos relatos descreve formação de aneurisma micótico nos ramos da artéria pulmonar em casos com evolução prolongada, fato que não ocorreu nesta paciente.

O cateterismo cardíaco nesta doença deve ser realizado para verificar a presença de aneurismas micóticos, sempre que o ECO não demonstrar esta complicação, apesar do risco potencial de embolia pulmonar durante a intervenção ${ }^{4}$.

Nosso caso ainda ilustra a malignidade da doença quando o foco de infecção não é ressecado, persistindo como fonte embolígena e causa de insucesso terapêutico.

Devem ser realizados exames adequados em pacientes com febre prolongada e, principalmente, obter-se a visibilização da artéria pulmonar pelos planos ecocardiográficos supraesternais em todos estes pacientes, principalmente em crianças com antecedentes de doença cardíaca congênita.

\section{Referências}

1. SanDretto MA, Scanlon GT - Multiple mycotic pulmonary artery aneurysms secondary to intravenous drug abuse. AJR 1984; 142: 89-90.

2. Singer-Jordan JR - Mycotic aneurysm of the pulmonary artery complicating ventriculoatrial shunt. AJR 1980; 135: 378-80.

3. Westaby S, Tan C, Foale R, Hallidie-Smith KA, Bentall HH-Mycotic aneurysm of the pulmonary artery. Direct surgical approach with preservation of lung tissue. J Cardiovasc Surg 1987; 28: 596-8.

4. Vargas-Barron J, Attie F, Buendia-Hernandez A, Keirns C, Esquivel-Avila J Echocardiographic recognition of pulmonary artery endarteritis in patent ductus arteriosus. Am Heart J 1985; 109: 368-70.

5. Vargas-Barron J, Avila-Rosales L, Romero-Cárdenas A, Rijlaarsdam M, Keirns
C, Buendia-Hernandez A - Echocardiographic diagnosis of a mycotic aneurysm of the main pulmonary artery and patent ductus arteriosus. Am Heart J 1992; 123 : 1707-9.

6. Touze JE, Mardelle T, Chauvet J et al - Les aneurysmes pulmonaires au cours de la persistance du canal arterial. Arch Mal Coeur 1985; 78: 1955-8.

7. Rowley KM, Clubb KS, Smith GJW, Cabin HS - Right-sided infective endocarditis as a consequence of flow-directed pulmonary-artery catheterization: a clinicipathological study of 55 autopsied patients. NEngl J Med 1984; 311: 1152-6.

8. Mansur AJ, Grinberg M, Gallucci SDD, Bellotti G, Jatene A, Pileggi F Endocardite infecciosa: análise de 300 episódios. Arq Bras Cardiol 1990; 54 : $13-21$. 